\title{
Helminth Infection Increases the Probability of Indeterminate QuantifERON Gold in Tube Results in Pregnant Women
}

\author{
Dawit Gebreegziabiher, ${ }^{1}$ Kassu Desta, ${ }^{2}$ Rawleigh Howe, ${ }^{3}$ and Markos Abebe ${ }^{3}$ \\ ${ }^{1}$ Mekelle University, Mekelle, Ethiopia \\ ${ }^{2}$ Addis Ababa University, Addis Ababa, Ethiopia \\ ${ }^{3}$ Armauer Hansen Research Institute (AHRI), Addis Ababa, Ethiopia
}

Correspondence should be addressed to Dawit Gebreegziabiher; dawit20081@gmail.com

Received 30 November 2013; Accepted 10 January 2014; Published 19 February 2014

Academic Editor: Umesh Gupta

Copyright (C) 2014 Dawit Gebreegziabiher et al. This is an open access article distributed under the Creative Commons Attribution License, which permits unrestricted use, distribution, and reproduction in any medium, provided the original work is properly cited.

Background. Approximately one-third of the world population is infected with M. tuberculosis and helminths (Kariminia et al. (2009), Walson et al. (2010)). Pregnancy and Helminth infection are known to suppress the $\mathrm{T}_{\mathrm{H}} 1$ response (Kariminia et al. (2009), Elias et al. (2006)) on which the QuantiFERON Gold in Tube (QFT-GIT) assay, that measures the released IFN- $\gamma$ upon in vitro stimulation with mycobacterial antigens, relies on (Thomas et al. (2010)). Objective. To determine whether QFT-GIT indeterminate result is significantly associated with helminth infection or not. Methods. In this cross-sectional study, eighty-five pregnant mothers were screened for parasitic and LTBI using Kato-Katz and QFT-GIT test-respectively, Result. The prevalence of helminth infection in pregnant mothers was $23(27 \%)$ of this $17(20 \%)$ was due to Schistosoma mansoni. Among the total of 85 study participants 26.8\% were QFT-GIT positive and 14 (17\%) had indeterminate results. Three samples $(21.4 \%)$ were randomly selected from the indeterminate QFT-GIT results and retested to check the reproducibility of the assay and remained indeterminate. QFT-GIT indeterminate result showed significant association with helminth infection. Conclusion. Helminth infections were significantly associated with indeterminate QFT-GIT results in pregnant mothers. Therefore further study is important to evaluate the possible effect of helminth infection by excluding the effect of pregnancy, as pregnancy also downregulates cellular immunity.

\section{Introduction}

Tuberculosis (TB) is a leading global cause of morbidity and mortality [1-4]. About 5\% of the M. tuberculosis infected individuals develop LTBI. This large pool of individuals with latent infection poses a major hurdle for global tuberculosis control efforts $[5,6]$. Active TB results from either uncontrolled primary infection or reactivation of LTBI particularly in young children, pregnant mothers, and immunecompromised individuals $[4,5,7,8]$.

Early identification and treatment of LTBI is a proposed key strategy for global TB prevention and control. However, the lack of a gold standard test to detect LTBI makes accurate diagnosis difficult [9]. Given the delay and difficulties in reaching a microbiological diagnosis of $\mathrm{TB}$ disease and the lack of positive microbiology in LTBI, the diagnosis of TB infection is mostly reliant on the demonstration of immunological memory cells to mycobacterial antigens $[7,10]$. Until whole blood assay such as Interferon-gamma release assay is developed, the only tool available to screen LTBI was the tuberculin skin test (TST) [11-13]. Nowadays, other in vitro T cell based assays for the diagnosis of LTBI are available. QFTGIT assay is one of these assays which measures the released IFN- $\gamma$ by sensitized T cells with specific M.TB antigens [9, 10, 12-15]. QFT-GIT is more specific than TST because it uses antigens which are encoded within region of difference 1 (early secreted antigenic target, $6 \mathrm{kDa}$ (ESAT-6), and culture filtrate protein, $10 \mathrm{kDa}(\mathrm{CFP}-10))$ and region of difference 11 (TB7.7) [10].

The prevalence of TB and helminth infection is geographically overlapping. Helminth infection is one of the factors that downregulates $\mathrm{T}_{\mathrm{H}} 1$ type immune response [16]. 
This $\mathrm{T}_{\mathrm{H}} 1$ to $\mathrm{T}_{\mathrm{H}} 2$ shift shows significant effect on $\mathrm{T}$ cell based immunological assays [9]. QFT-GIT detects response of effector $\mathrm{T}$ cells that have recently encountered antigens, though the presence of noninterpretable result, especially in developing countries, reduces its usefulness [16, 17]. Very few recent studies reported that helminth infections significantly associated with indeterminate QFT-GIT results [9], and this was also observed in this study.

\section{Methods}

A cross-sectional design was used to determine whether the indeterminate QFT-GIT assay is significantly associated with helminth infection or not. The study was conducted in Mekelle, Tigray Regional State, Northern Ethiopia, from October 2011 to July 2012. Consecutive samples were collected from 85 voluntary pregnant mothers at the last week of their ninth month of pregnancy from $\mathrm{MCH}$ Departments of Mekelle Hospital, Semen Health Center, and Ayder Referral Hospital. Based on the helminth and latent TB infections, our study participants were grouped into helminth and LTBI positive, helminth and LTBI negative, helminth positive and LTBI negative, and helminth negative and LTBI positive.

2.1. Parasitological Examination. Stool samples were collected in screw capped container and duplicate Kato slides were prepared within one hour of collection and examined within two days of collection. Wet mount preparation was also done to evaluate for hook worm and protozoan infection within 30 minutes. Following delivery, all mothers who were positive for any species of parasites were treated accordingly.

2.2. QuantiFERON-TB Gold In Tube Assay. All study participants were screened for LTBI using QFT-GIT, Cellestis Limited, Carnegie, Victoria, Australia. One milliliter of blood was collected into each of the three heparinized tubes: the nil (grey capped) negative control (containing only heparin), the mitogen (purple) positive control (containing phytohemagglutinin), and antigens (red capped) tube that contains $M$. tuberculosis specific antigens (ESAT-6, CFP-10, and TB7.7). The tubes were well mixed to ensure the appropriate contact of blood with antigens or mitogen and incubated at $37^{\circ} \mathrm{C}$ for 20 hours. Plasma was harvested by centrifuging at $3000 \mathrm{rpm}$ for 15 minutes and stored at $-80^{\circ} \mathrm{C}$. Plasma was thawed and ELISA was performed according to the QFT-GIT kit protocol and the optical densities (OD) were measured using a microplate ELISA reader (Molecular Devices Corporation, USA) fitted with a $450 \mathrm{~nm}$ and $650 \mathrm{~nm}$ filter. The actual IFN$\gamma$ concentration in the sample was calculated based on the curve generated from the standards using QuantiFERONTB Gold Analysis Software supplied by Cellestis Limited, Carnegie, Victoria, Australia.

The assay result was positive if the net IFN- $\gamma$ response to the TB antigens was $\geq 0.35 \mathrm{IU} / \mathrm{mL}$, regardless of the mitogen response, and negative if the net IFN- $\gamma$ response was $<0.35 \mathrm{IU} / \mathrm{mL}$ and the sufficient net mitogen response $(>0.5 \mathrm{IU} / \mathrm{mL})$. A result is said to be indeterminate if there was excessive IFN- $\gamma$ production from the negative control $(>8.0 \mathrm{IU} / \mathrm{mL})$ or insufficient net IFN- $\gamma$ response from mitogen $(<0.5 \mathrm{IU} / \mathrm{mL})$ plus net $\mathrm{TB}$ antigen response $(<0.35 \mathrm{IU} / \mathrm{mL})$.

2.3. Statistical Analysis. Data was entered and analyzed using the SPSS version 19. Descriptive statistics for sociodemographic and clinical characteristic was performed. Univariate logistic regression was used to explore the association between QFT-GIT indeterminate result and difference clinical data. Chi square $\left(\chi^{2}\right)$ test was also used to compare QFT-GIT indeterminate results with helminth positive and negative participants.

2.4. Ethical Consideration. Ethical approval was obtained from the Department of Medical Laboratory Sciences (MLS), College of Allied Health Sciences (CAHS), Addis Ababa University (AAU), and Armauer Hansen Research Institute (AHRI). Participants were enrolled in the study after they adequately understood the purpose of the study and signed in the written informed consent.

\section{Result}

3.1. Sociodemographic Characteristic. The median age of the study participants was 25 years (IQR 22, 28). Educational background of the study participants was primary school $50.6 \%$, secondary school $28.2 \%$, tertiary $10.6 \%$, and illiterates $10.6 \%$. All of the study participants were urban residents. Majorities (83.5\%) of study participants were housewives and $11.8 \%$ were government employees.

3.2. Clinical Information. Forty-seven percent of the study participants had their first pregnancy, 29.41\% second, and $23.5 \%$ third and above pregnancies. Of the total participants, only $15.29 \%$ were reported as BCG vaccinated. BCG scar was found only in $23.1 \%$ from the vaccinated participants. Only one participant had previous TB history eight years ago and five had previous contact with TB patients within the last 10 years. Only five of the study participants had close contact with TB patient 5 to 10 years ago (Table 1).

3.3. Parasitological Infection. The prevalence of helminth infection was $23(27.06 \%)$ and the most prevalent helminths among the study participants were Schistosoma mansoni 17 (20\%) followed by Ascaris lumbricoides 13 (15.3\%), Enterobius vermicularis 8 (9.4\%), Trichuris trichiura 7 (8.2\%), and Hymenolepis nana 5 (5.8\%). The prevalence of protozoaninfection was $7(8.33 \%)$, of this $6(7.24 \%)$ and $2(2.35 \%)$ was account to Entamoeba histolytica/dispar and Giardia lamblia, respectively. None of the study participants were found positive for Schistosoma haematobium. The overall intestinal parasite prevalence was 24 (28.2\%). Among the helminth positive study participants, $31.64 \%$ had double or triple infection. Logistic regression was performed for age categories, occupation, level of educational background, and the number of pregnancies, and a significant association was observed only for educational level and parasitic infection. As the level of education increases from illiteracy to primary 
TABLE 1: Association of indeterminate QFT-GIT results with clinical data of study participants $(N=85)$.

\begin{tabular}{lcc}
\hline Characteristic & Freq. $(\%)$ & OR (95\% CLI) \\
\hline Number of pregnancies & & \\
$\quad$ First & $40(47.5)$ & Reference \\
Second & $25(29.4)$ & $0.23(-0.03-3.09)$ \\
3rd and above & $20(23.5)$ & $0.11(-0.65-7.01)$ \\
BCG vaccination & & \\
Yes & $13(15.3)$ & Reference \\
No & $72(84.7)$ & $0.09(-0.2-14.03)$ \\
BCG scar & $3(23.1)$ & Reference \\
Yes & $10(76.9)$ & $0.11(-0.75-18)$ \\
No & & \\
TB history & $1(1.18)$ & Reference \\
Yes & $84(98.82)$ & $0.82(-0.79-17)$ \\
No & & \\
TB contact & & Reference \\
Yes & $5(5.88)$ & $2.03(0.31-13.51)$ \\
No & $80(84.12)$ &
\end{tabular}

TB contact history ${ }^{* *}=$ contact in $5-10$ years.

and then to secondary level, the odds of parasitic infection were decreased $(\mathrm{OR}=0.50, \mathrm{CI}=0.211$ to 0.992 , and $P=0.048)$.

3.4. Latent Tuberculosis Infection. The result of QFT-GIT test indicated that LTBI was found in $26.83 \%$ while $17 \%$ had an indeterminate result. Three samples (21.4\%) were randomly selected from the indeterminate QFT-GIT results and retested to check the reproducibility of the test which showed the same result. All indeterminate results were due to an insufficient IFN- $\gamma$ response to mitogen. Fisher's exact test correlation coefficient was performed to explore relationship between QFT-GIT indeterminate result and helminth infection. QFT-GIT indeterminate result and helminth infection showed positive relationship ( $r=0.13, n=80$, and $P=0.036$ ). Although the majority of the BCG vaccinated participants (76.92\%) were QFT-GIT negative, $15.38 \%$ and $7.69 \%$ had positive and indeterminate QFT-GIT results, respectively. Only $20 \%$ of the study participants who had BCG scar were positive for QFT-GIT. Only 6\% of the total participants were reported to have contact with TB patients within 3 to 5 years, and $60 \%$ them had positive QFT-GIT and the remained $40 \%$ had indeterminate QFT-GIT results. Associations of QFTGIT positivity with BCG vaccination, BCG scar, and contact with active TB patients were assessed by univariate logistic regression. The risk of LTBI was higher in participants who had contact with $\mathrm{TB}$ patients $(\mathrm{OR}=2.03, \mathrm{CI}=0.31$ to 13.51 , and $P=0.046)$. BCG vaccination $(P=0.123)$, BCG scar $(P=0.52)$, previous TB history $(P=0.321)$, and pregnancy (0.409) showed no statistically significant association. The proportion of QFT-GIT indeterminate results were significantly higher in helminth positive, 10 (71.4\%), participants than helminth negative, 4 (28.6\%), with $\chi^{2}=6.06, d f=1$ and
$P=0.048$, but this was not observed in protozoan infection $(P=0.273)$.

\section{Discussion}

Helminth infections are highly prevalent in populations where Mycobacterium tuberculosis infection is also endemic. It has been widely conjectured that the strong mucosal $\mathrm{T}_{\mathrm{H}} 2$ and $\mathrm{T}$ regulatory cell responses elicited by these parasites can downregulate protective immune response against Mycobacterium tuberculosis [18].

This study was conducted to see if coinfection with helminth has impact on the use of QFT-GIT TB Gold assay in areas where both diseases are endemic. The prevalence of LTBI among pregnant women was $26.83 \%$ using QFTGIT. Community based LTBI prevalence study in Afar region, Ethiopia, was higher (63.7\%) [19] than our finding. Other studies in the same site reported that $59.3 \%$ of TB suspected participants were positive for LTBI, using QFT-GIT assay [20]. A study conducted at Addis Ababa University on male medical students also showed that $43.9 \%$ were QFTGIT positive which is higher than our result [21]. One possible explanation for this might be the effect of helminth coinfection [16] and pregnancy, because pregnancy decreases cell mediated immunity and also gender difference of study participants [22].

QFT-GIT has been recommended as an alternative assay to TST to screen LTBI. QFT-GIT has better specificity and is not affected by BCG vaccination and other non- $M$. tuberculosis species infection because it uses $M$. tuberculosis specific antigens (ESAT-6/CFP-10 and TB7.7). Therefore, QFT-GIT is being used to screen contacts of TB patients to check whether contacts infected by MTB or not. Our data also showed that contacts had increased risk for LTBI $(\mathrm{RR}=2.3, P=0.046)$. The proportion of QFT-GIT positivity had no statistically significant association with previous $B C G$ vaccination $(P=$ $0.123)$ and BCG scar $(P=0.52)$ using univariate logistic regression. In contrast to our study, a research conducted in Afar region, Ethiopia reported that QFT-GIT positivity was higher in BCG vaccinated than non-vaccinated participants (76.0\% versus $61.2 \%$ ) [19]. A study conducted in Norway also indicated that $\mathrm{TB}$ exposure $(\mathrm{OR}=1.59)$ and previous $\mathrm{TB}$ disease $(\mathrm{OR}=11.60)$ had association with QFT-GIT positivity but not with BCG vaccination [23].

One of the potential drawbacks of QFT-GIT assay is the presence of indeterminate results which limits interpretation. Indeterminate results can happen due to different factors one of which is chronic helminth infection [9]. In our result, $17 \%$ of the study participants had indeterminate QFT-GIT. In addition, all of the study participants who had indeterminate QFT-GIT result were due to low or failure of cells to respond to mitogen (positive control). This was explained by effect of chronic helminth infection on the $\mathrm{T}_{\mathrm{H}} 1$ IFN- $\gamma$ response as helminth infection and QFT-GIT indeterminate result showed significant positive association $(r=0.13, P=$ 0.036). A study conducted in Australia showed that $11 \%$ of study participants had QFT-GIT indeterminate result and all were due to the failure to respond to the positive 
control: the majority of them were helminth positive [7]. Another study conducted in Bangladesh also indicated that above $95 \%$ of the study participants who had indeterminate QFT-GIT results were due to insufficient response to mitogen and those were associated with helminth infection [9].

\section{Conclusion}

TB and helminth infections, which induce antagonizing immune response, are epidemiologically overlapping in developing countries; and thus helminth infection might be further complicating TB control and prevention programs by attenuating $\mathrm{T}_{\mathrm{H}} 1$ wings of the host immune system. Since QFT-GIT assay is also based on IFN- $\gamma$ response from $\mathrm{T}_{\mathrm{H}} 1 \mathrm{~T}$ cells in response to MTB antigens stimulation, the sensitivity of the assay is compromised by helminth infection. In this study, significant proportion of QFT-GIT results were categorized as indeterminate and also significantly associated with the helminth infection. Thus special consideration is required during interpretation of QFT-GIT indeterminate results in areas where helminth infection is common.

\section{Conflict of Interests}

The authors declare that there is no conflict of interests regarding the publication of this paper.

\section{Acknowledgment}

The authors would like to acknowledge the financial support of Sida/NORAD through AHRI core budget.

\section{References}

[1] J. Lighter-Fisher and A. M. Surette, "Performance of an interferon-gamma release assay to diagnose latent tuberculosis infection during pregnancy," Obstetrics \& Gynecology, vol. 119, pp. 1088-1095, 2012.

[2] Z. Bentwich, R. Horner, and G. Borkow, "De-worming in developing countries as a feasible and affordable means to fight co-endemic infectious diseases," Open Biology Journal, vol. 3, pp. 97-103, 2010.

[3] C. D. Bourke, R. M. Maizels, and F. Mutapi, "Acquired immune heterogeneity and its sources in human helminth infection," Parasitology, vol. 138, no. 2, pp. 139-159, 2011.

[4] F. G. Sheriff, K. P. Manji, M. P. Manji et al., "Latent tuberculosis among pregnant mothers in a resource poor setting in Northern Tanzania: a cross-sectional study," BMC Infectious Diseases, vol. 10 , article 52, 2010.

[5] O. M. Loto and I. Awowole, "Tuberculosis in pregnancy: a review," Journal of Pregnancy, vol. 2012, Article ID 379271, 7 pages, 2012.

[6] R. N. Van Zyl-Smit, M. Pai, K. Peprah et al., "Within-subject variability and boosting of $\mathrm{t}$-cell interferon- $\gamma$ responses after tuberculin skin testing," American Journal of Respiratory and Critical Care Medicine, vol. 180, no. 1, pp. 49-58, 2009.

[7] S. Banfield, E. Pascoe, A. Thambiran, and A. D. B. Siafarikas, "Factors associated with the performance of a blood-based interferon- $\gamma$ release assay in diagnosing tuberculosis," PLoS One, vol. 7, no. 6, Article ID e38556, 2012.

[8] G. Hassan, W. Qureshi, and S. M. Kadri, "Congenital tuberculosis," JK Science, vol. 8, no. 4, 2006.

[9] T. A. Thomas, D. Mondal, Z. Noor et al., "Malnutrition and helminth infection affect performance of an interferon $\gamma$-release assay," Pediatrics, vol. 126, no. 6, pp. e1522-e1529, 2010.

[10] I. Latorre, X. Martínez-Lacasa, R. Font et al., "IFN- $\gamma$ response on T-cell based assays in HIV-infected patients for detection of tuberculosis infection," BMC Infectious Diseases, vol. 10, article 348, 2010.

[11] D. Menzies, "What does tuberculin reactivity after bacille Calmette-Guérin vaccination tell us?" Clinical Infectious Diseases, vol. 31, no. 3, pp. S71-S74, 2000.

[12] I. Brock, M. E. Munk, A. Kok-Jensen, and P. Andersen, "Performance of whole blood IFN- $\gamma$ test for tuberculosis diagnosis based on PPD or the specific antigens ESAT-6 and CFP-10," International Journal of Tuberculosis and Lung Disease, vol. 5, no. 5, pp. 462-467, 2001.

[13] M. Pai, R. Joshi, S. Dogra et al., "Persistently elevated T cell interferon- $\gamma$ responses after treatment for latent tuberculosis infection among health care workers in India: a preliminary report," Journal of Occupational Medicine and Toxicology, vol. 1, no. 1, article 7, 2006.

[14] G. H. Mazurek, J. Jereb, P. Lobue, M. F. Iademarco, B. Metchock, and A. Vernon, "Guidelines for using the QuantiFERON-TB Gold test for detecting Mycobacterium tuberculosis infection, United States," Morbidity and Mortality Weekly Report, vol. 54, no. 15, pp. 49-55, 2005.

[15] B. W. Laartz, H. J. Narvarte, D. Holt, J. A. Larkin, and W. F. Pomputius III, "Congenital tuberculosis and management of exposures in a neonatal intensive care unit," Infection Control and Hospital Epidemiology, vol. 23, no. 10, pp. 573-579, 2002.

[16] A. Kariminia, Z. Sharifnia, A. Aghakhani et al., "Comparison of quantiFERON TB-G-test to TST for detecting latent tuberculosis infection in a high-incidence area containing BCG-vaccinated population," Journal of Evaluation in Clinical Practice, vol. 15, no. 1, pp. 148-151, 2009.

[17] K. Shanaube, J. Hargreaves, K. Fielding et al., "Risk factors associated with positive quantiFERON-TB gold in-tube and tuberculin skin tests results in Zambia and South Africa," PLoS One, vol. 6, no. 4, Article ID e18206, 2011.

[18] J. A. Potian, W. Rafi, K. Bhatt, A. McBride, W. C. Gause, and P. Salgame, "Preexisting helminth infection induces inhibition of innate pulmonary anti-tuberculosis defense by engaging the IL4 receptor pathway," Journal of Experimental Medicine, vol. 208, no. 9, pp. 1863-1874, 2011.

[19] M. Legesse, G. Ameni, G. Mamo, G. Medhin, G. Bjune, and F. Abebe, "Community-based cross-sectional survey of latent tuberculosis infection in Afar pastoralists, Ethiopia, using QuantiFERON-TB Gold In-Tube and tuberculin skin test," BMC Infectious Diseases, vol. 11, article 89, 2011.

[20] M. Legesse, G. Ameni, G. Mamo, G. Medhin, G. Bjune, and F. Abebe, "Performance of QuantiFERON-TB Gold In-Tube (QFTGIT) for the diagnosis of Mycobacterium tuberculosis (Mtb) infection in Afar Pastoralists, Ethiopia," BMC Infectious Diseases, vol. 10, article 354, 2010.

[21] A. F. Dagnew, J. Hussein, M. Abebe et al., "Diagnosis of latent tuberculosis infection in healthy young adults in a country with high tuberculosis burden and BCG vaccination at birth," BMC Research Notes, vol. 5, no. 1, article 415, 2012. 
[22] G. Reinhard, A. Noll, H. Schlebusch, P. Mallmann, and A. V. Ruecker, "Shifts in the TH1/TH2 balance during human pregnancy correlate with apoptotic changes," Biochemical and Biophysical Research Communications, vol. 245, no. 3, pp. 933938, 1998.

[23] A. M. Dyrhol-Riise, G. Gran, T. Wenzel-Larsen, B. Blomberg, C. G. Haanshuus, and O. Mørkve, "Diagnosis and followup of treatment of latent tuberculosis; the utility of the QuantiFERON-TB Gold In-tube assay in outpatients from a tuberculosis low-endemic country," BMC Infectious Diseases, vol. 10, article 57, 2010. 


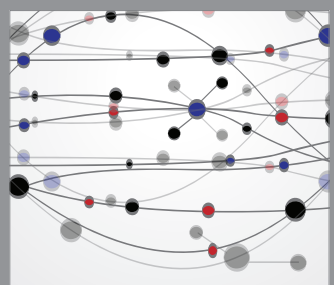

The Scientific World Journal
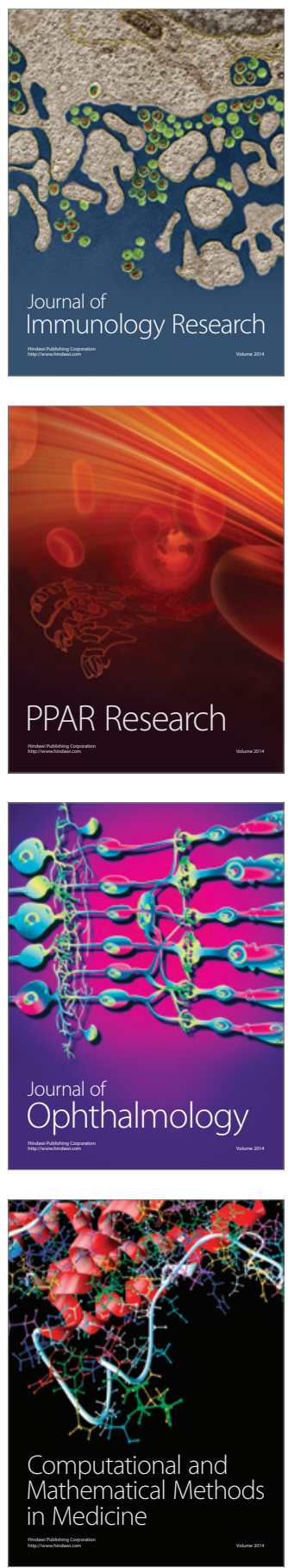

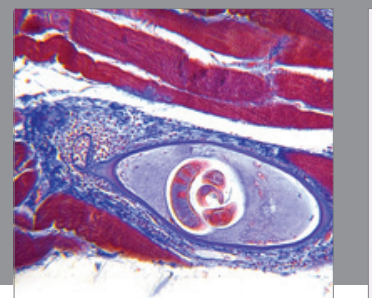

Gastroenterology

Research and Practice
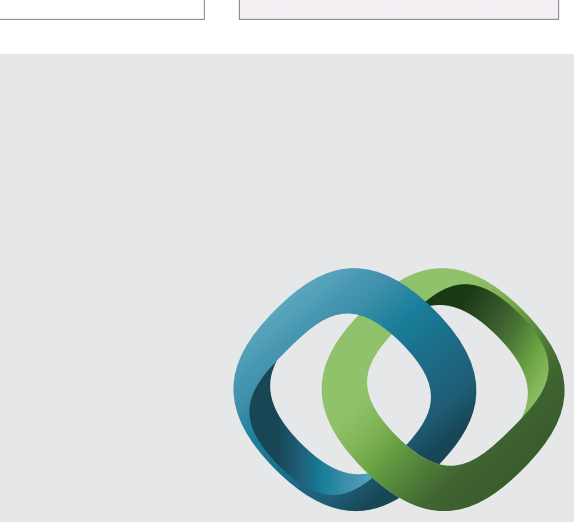

\section{Hindawi}

Submit your manuscripts at

http://www.hindawi.com
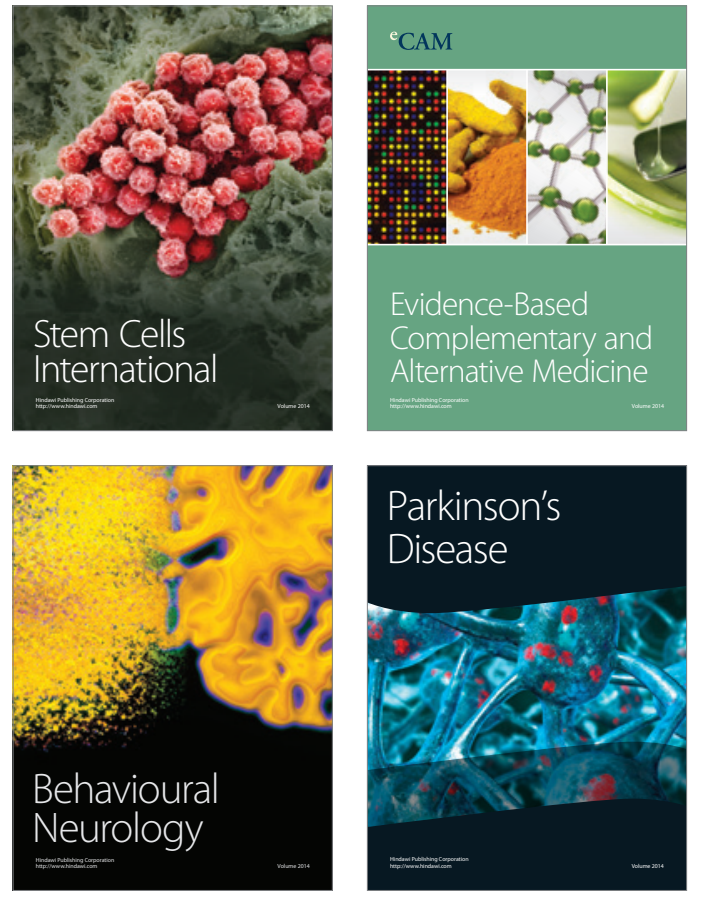
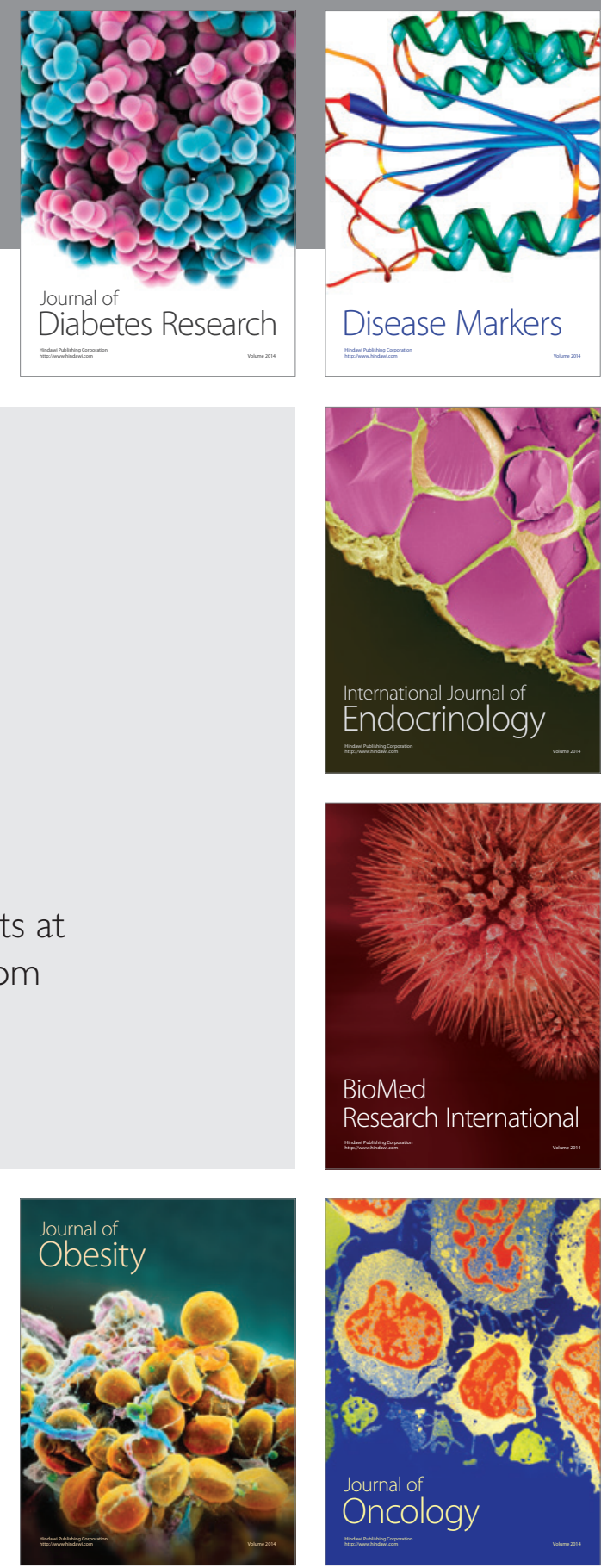

Disease Markers
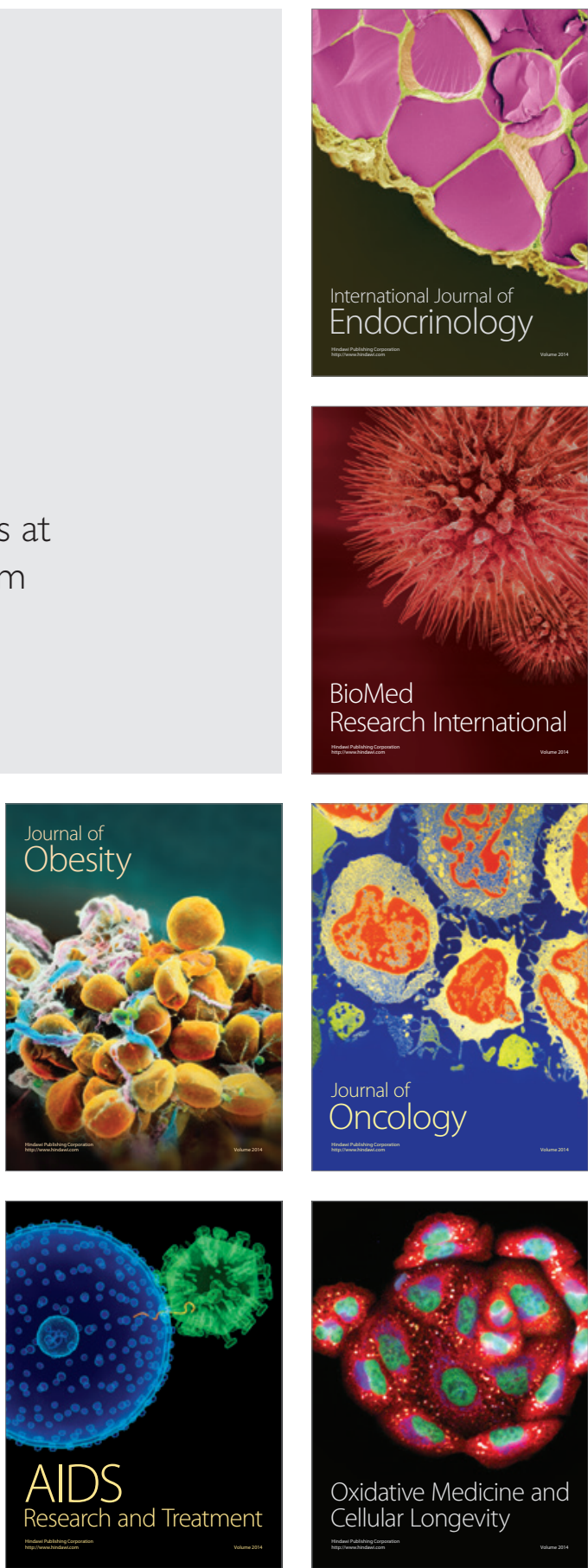